\title{
Electrical characterization of MgO tunnel barriers grown on InAs (001) epilayers
}

\author{
F. Magnus, S. K. Clowes, A. M. Gilbertson, W. R. Branford, \\ E. D. Barkhoudarov, and L. F. Cohen ${ }^{\text {a) }}$ \\ Blackett Laboratory, Imperial College, Prince Consort Road, London SW7 2AZ, United Kingdom \\ L. J. Singh, Z. H. Barber, and M. G. Blamire \\ Department of Materials Science and Metallurgy, University of Cambridge, Pembroke Street, \\ Cambridge CB2 3QZ, United Kingdom \\ P. D. Buckle, L. Buckle, and T. Ashley \\ QinetiQ, Malvern Technology Centre, St. Andrews Road, Malvern WR14 3PS, United Kingdom \\ D. A. Eustace and D. W. McComb \\ Department of Materials, Imperial College, London SW7 2AZ, United Kingdom
}

(Received 27 June 2007; accepted 22 August 2007; published online 20 September 2007)

\begin{abstract}
The authors examine the electrical properties of ultrathin $\mathrm{MgO}$ barriers grown on (001) InAs epilayers and the dependence on InAs surface pretreatment and growth conditions. Pretreatment improves the yield of tunnel junctions and changes the roughness of the interface between oxide and semiconductor. Electrical characterization confirms that tunnel barriers with appropriate values of interface resistance for efficient spin injection/detection have been achieved. Using the Rowell criteria and various tunneling models, the authors show that single step tunneling occurs above $150 \mathrm{~K}$. Incorporating a thermal smearing model suggests that tunneling is the dominant transport process down to $10 \mathrm{~K}$. (C) 2007 American Institute of Physics. [DOI: 10.1063/1.2784933]
\end{abstract}

Control of spin in semiconductors remains highly topical. ${ }^{1-3}$ Although realizing the spin transistor ${ }^{4}$ or spin lifetime transistor ${ }^{5}$ has proved to be very difficult, certain concepts have now been shown to be robust and promising. ${ }^{3}$ Optimizing the results requires proper design of interfaces so that polarization is maintained efficiently across interfaces. Significant injection of spin from ferromagnetic (FM) metals into semiconductors $(\mathrm{S})$ has been achieved for spin lightemitting diodes and it was with these devices that the need for a tunnel barrier injector ${ }^{6-8}$ was confirmed. Narrow gap semiconductors (NGSs) are attractive because of high electron mobility and strong spin-orbit coupling. Building compatible tunnel barriers between NGS and FM metals remains a challenge, particularly if one is to exploit the spin filtering properties of $\mathrm{MgO}$ in $\mathrm{FM} / \mathrm{oxide} / \mathrm{S}$ structures. ${ }^{9} \mathrm{MgO}$ is also attractive because of its low barrier height. ${ }^{10,11}$ To harness the advantage offered by $\mathrm{MgO}$, we must understand the electrical properties of $\mathrm{MgO}$ barriers grown on NGS which are as yet unexplored. Consequently, we have carried out a detailed study to determine the electrical integrity of structurally well characterized $^{12} \mathrm{MgO}$ barriers with a specific aim to understand the influence of surface treatment and growth conditions. Importantly, we find that single step tunneling occurs through these barriers above $150 \mathrm{~K}$ rendering them effective for efficient injection or detection of spin. Moreover, taking thermal smearing into account indicates that tunneling is also the dominant transport process at low temperatures.

Thin $\mathrm{MgO}$ layers were deposited ex situ onto $1 \mu \mathrm{m}$ thick epilayers of $\operatorname{InAs}(001) / \mathrm{GaAs}$ grown by molecular beam epitaxy. One of three chemical surface treatments was carried out on the InAs prior to $\mathrm{MgO}$ deposition: (i) degrease in acetone and isopropanol, (ii) $18.5 \% \mathrm{HCl}$ etch and $2.1 \%$

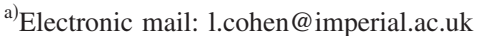

$\left(\mathrm{NH}_{4}\right)_{2} \mathrm{~S}$ for surface passivation (single etch), (iii) recipe (ii) performed twice (double etch). The $\mathrm{MgO}$ was grown by reactive sputtering from a $\mathrm{Mg}$ target in an $\mathrm{Ar}-30 \% \mathrm{O}_{2}$ gas mixture. The substrate temperature during the $\mathrm{MgO}$ growth was 100 or $200{ }^{\circ} \mathrm{C}$. After $\mathrm{MgO}$ growth, a $20 \mathrm{~nm}$ thick film of Co was deposited. Details of the growth and structural properties are given in Ref. 12.

The three Rowell criteria ${ }^{13}$ are commonly employed to show that tunneling is dominant: (i) The barrier current decays exponentially with increasing insulator thickness. (ii) The conductance spectrum $G(V)$ is nonlinear, as described by the Brinkman-Dynes-Rowell ${ }^{14}$ (BDR) model. (iii) The temperature dependence of the zero bias conductance $G_{0}(T)$ is weakly insulatorlike, as described by the Stratton model. ${ }^{15}$ However, recent studies have shown that criteria (i) and (ii) alone are not sufficient to rule out pinholes in a magnetic tunnel junction (MTJ). ${ }^{16}$ Furthermore, tunnel junctions can exhibit a sharp dip in conductance around zero bias. ${ }^{17,18}$ This is know as the zero bias anomaly (ZBA) and is usually attributed to resonant multistep tunneling via impurities which is not treated by the above models.

The BDR model is conveniently approximated at low bias voltages by a second order polynomial yielding three independent fitting parameters. These are generally taken to be the barrier thickness $d$, the mean barrier height $\varphi$, and the barrier asymmetry $\Delta \varphi$. The BDR expression can be found in Ref. 14, Eq. (7). The Stratton model can give two independent parameters, generally $d$ and $\varphi$, and is given in convenient form in Ref. 17, Eq. (5). Recently, however, Miller et al. have pointed out two severe shortcomings of WentzelKramers-Brillouin-based tunneling models such as the BDR and Stratton models. Firstly, barrier roughness is not taken into account. Due to the first Rowell criterion, thinner regions will dominate resulting in lower $d$ than expected and 
TABLE I. Summary of sample properties. $T_{g}$ is the growth temperature and $t$ is the oxide thickness determined by TEM with $\pm 0.3 \mathrm{~nm}$ error.

\begin{tabular}{llcccc}
\hline \hline Sample & Pretreatment & $T_{g}\left({ }^{\circ} \mathrm{C}\right)$ & $t(\mathrm{~nm})$ & $r_{b}\left(\Omega \mathrm{m}^{2}\right)$ & Tunneling \\
\hline $21472-1$ & Degreased & 100 & 1.8 & $7.54 \times 10^{-10}$ & no \\
$21487-1$ & Degreased & 200 & 1.8 & $1.13 \times 10^{-10}$ & no \\
$21527-1$ & Degreased & 200 & 1.8 & $2.4 \times 10^{-9}$ & yes \\
$21358-2$ & Single etch & 200 & 1.8 & $3.5 \times 10^{-9}$ & yes \\
21424 & Single etch & 200 & 1.2 & $1.75 \times 10^{-9}$ & yes \\
$21472-2$ & Double etch & 100 & 1.8 & $2.47 \times 10^{-9}$ & yes \\
\hline \hline
\end{tabular}

greater $\varphi \cdot{ }^{19}$ Secondly, the routinely employed expressions for the BDR and Stratton models use the free electron mass and do not take into account the effective electron mass $m^{*}$ in the barrier material or conductor. ${ }^{20} \mathrm{In}$ a $\mathrm{Co} / \mathrm{MgO} / \mathrm{InAs}$ structure $m^{*}$ varies by almost two orders of magnitude as $\mathrm{m}^{*} / \mathrm{m}_{e}=0.026$ in InAs. ${ }^{21}$

To obtain significant spin injection and detection, the interface resistance $r_{b}$ needs at least to satisfy the condition $r_{b} \approx r_{N}=\rho_{N} l_{\text {sf }}$ where $\rho_{N}$ is the resistivity and $l_{\text {sf }}$ the spin diffusion length of the nonmagnetic material. ${ }^{2,6}$ The spin diffusion length in the nondegenerate limit can be defined as $\left(k_{B} T \mu \tau_{\mathrm{sf}} / 2 e\right)^{1 / 2}$ where $\mu$ is the mobility and $\tau_{\mathrm{sf}}$ is the spin lifetime of the carriers. From a knowledge of the spin lifetime in bulk InAs, ${ }^{22}$ we can estimate the value of $r_{b} \approx 10^{-9}-10^{-10} \Omega \mathrm{m}^{2}$ at room temperature. A direct interface between gold and InAs yields ${ }^{23}$ an interface resistance of $\sim 10^{-12} \Omega \mathrm{m}^{2}$ and this leaves scope for growing a barrier material on the InAs to reach the desired $r_{b}$. From electrical characterization of MTJs, MgO tunnel barriers ${ }^{10,11}$ appear to have a relatively low barrier height of $0.9 \mathrm{eV}$. This allows us to predict, using the Simmons model, ${ }^{24}$ that to achieve the desired $r_{b}$ the target thickness of $\mathrm{MgO}$ should be $\sim 1.3 \mathrm{~nm}$ on InAs. Table I shows a summary of the values for our samples with $r_{b}$ varying from $3.5 \times 10^{-9}$ to 1.1 $\times 10^{-10} \Omega \mathrm{m}^{2}$ which is in the desired range for efficient spin injection and detection in a $\mathrm{Co} / \mathrm{MgO} / \mathrm{InAs}$ structure.

Conductance properties vary substantially but all surface preparation recipes produce junctions which show signs of tunneling. Figure 1 shows typical tunnelinglike $G(V)$ spectra from $T=10-300 \mathrm{~K} . G(V)$ is parabolic at low $V$ and high $T$, but below $\sim 200 \mathrm{~K}$ a sharp dip in conductance appears to emerge around zero bias. This ZBA is present in almost all our tunneling spectra regardless of growth temperature and InAs pretreatment. The BDR model can therefore only be applied to the high $T$ data and a fit to the $300 \mathrm{~K}$ curve is shown in the figure.

The right inset to Fig. 1 shows the temperature dependence of $G_{0}(T)$ for several junctions on the same wafer as shown in the main graph. There is an excellent agreement with the Stratton model above $\sim 200 \mathrm{~K}$ and the temperature below which the ZBA emerges agrees with the full conductance curves. We find, in the high $T$ regime, both a BDR-like $G(V)$ and a Stratton-like $G_{0}(T)$ which is a strong indication that tunneling is the dominant transport mechanism in these junctions at high $T$.

A recent study has shown that the temperature dependence of the ZBA can be explained by including the effects of thermal smearing on the tunneling process. ${ }^{25}$ By taking the convolution of the low $T$ data $(10 \mathrm{~K})$ with a Gaussian function, ${ }^{26}$ we can simulate higher $T$ curves and obtain the effective smearing temperature $T^{*}$ required to reproduce Downloaded 03 Jun 2009 to 131.227.178.130. Redistribution subje

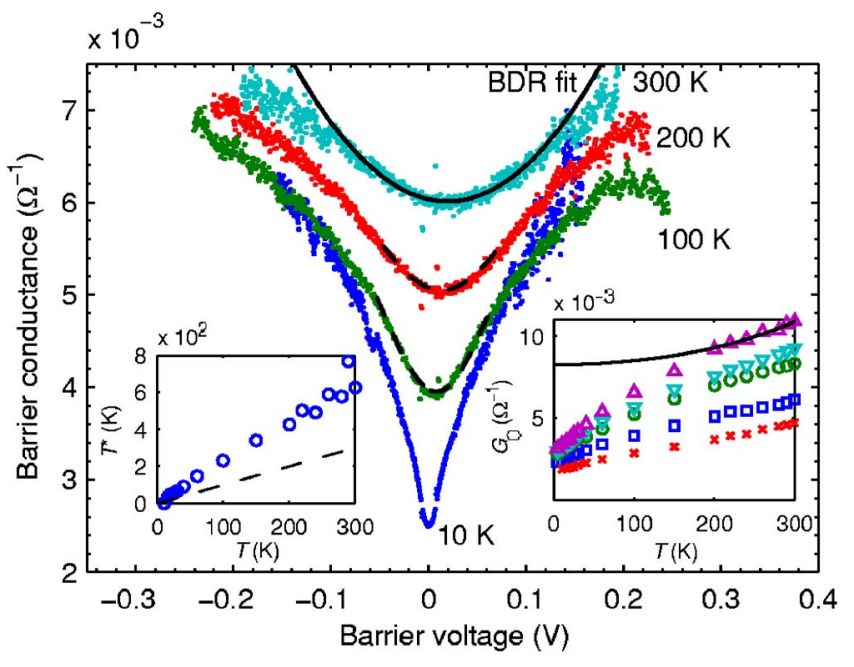

FIG. 1. (Color online) Typical barrier conductance spectra from room temperature down to $10 \mathrm{~K}$ showing parabolicity at high temperatures and the emergence of the ZBA at low temperatures. The solid line is a fit to the BDR model at $300 \mathrm{~K}$. The right inset shows the temperature dependence of the zero bias conductance for several junctions on the same wafer. The solid line is a fit to the Stratton model above $200 \mathrm{~K}$. The dashed lines in the main group show the effect of thermally smearing the $10 \mathrm{~K}$ data allowing for a small offset in $G$ and similarly a shift in $V$ [for $100 \mathrm{~K}(200 \mathrm{~K}) G_{\text {offset }}=6$ $\times 10^{-4}\left(1.1 \times 10^{-3}\right) \Omega^{-1}$ and $V_{\text {offset }}=6(12) \mathrm{mV}$. The left inset shows the smearing temperature vs actual temperature.

higher $T$ data, as shown by the dashed lines in Fig. 1. The left inset of Fig. 1 shows a $T^{*}$ to $T$ ratio of approximately 2 (the previous study finds a ratio of 1.6-2.0).

The absolute barrier parameters extracted from the fitting can only be treated as a guide to barrier properties due to the uncertainty in $\mathrm{m}^{*}$. We fix the effective mass to $\mathrm{m}^{*} / \mathrm{m}_{e}$ $=0.1$ as this gives reasonable values for the fitting parameters. Thus, despite the uncertainty about their absolute values, we can use the fitting parameters as a guide to compare electrical variability between junctions of the same materials.

A spread in $G_{0}$ (at fixed $T$ ) between different junctions on each wafer is observed because of a variation in thickness (first Rowell criterion). Figure 2 shows the relationship between $G_{0}$ at $300 \mathrm{~K}$ and the thickness $d$, as determined by fitting to the BDR model. The relationship is indeed close to

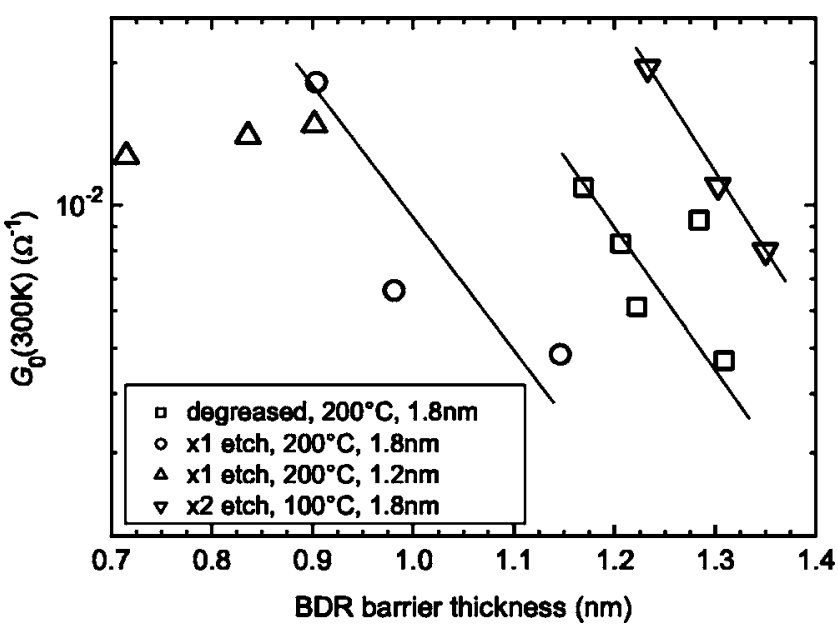

FIG. 2. Zero bias conductance at $300 \mathrm{~K}$ of each junction vs the barrier thickness as determined by fitting to the BDR model. The thicknesses in the legend are determined by TEM. The straight lines are a guide to the eye showing the expected exponential decay of $G_{0}$.

to AIP license or copyright; see http://apl.aip.org/apl/copyright.jsp 


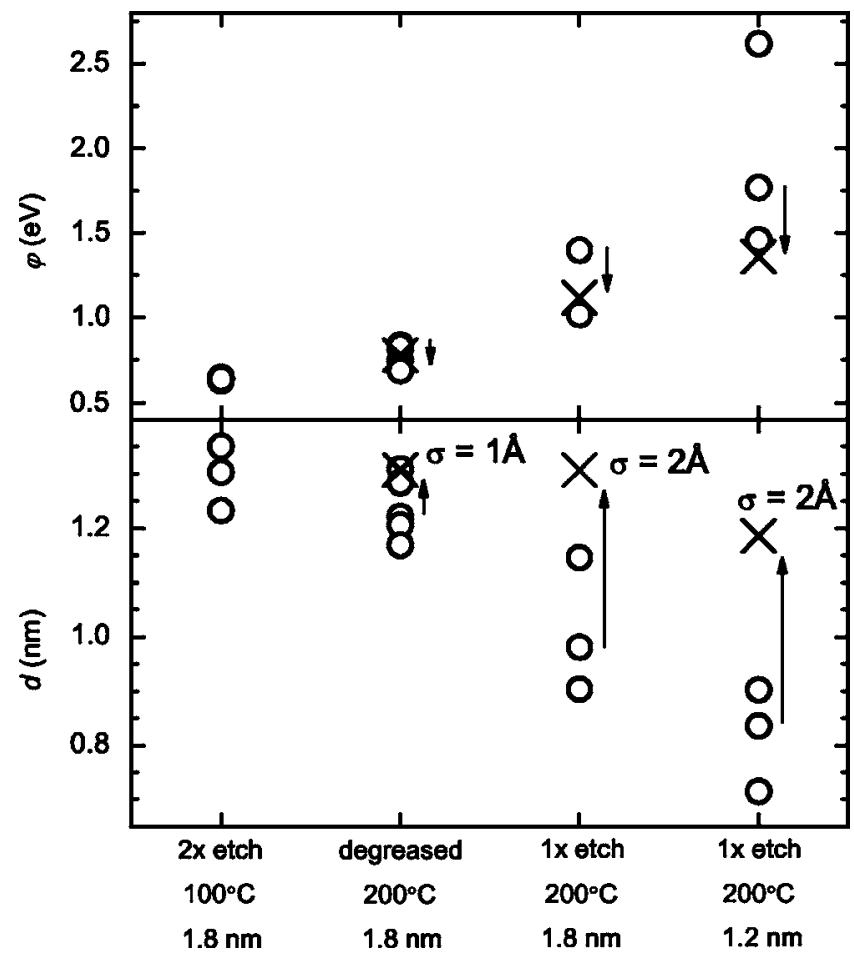

FIG. 3. Comparison of barrier thickness $d$ and height $\varphi$ determined by fitting to the BDR model for four different samples. The thickness determined by TEM is given below the $x$ axis. The crosses show how barrier roughness $\sigma$ can account for the apparent trend in $d$ and $\varphi$.

exponential within each wafer except for the wafer with the thinnest barrier. Interestingly, we find a discontinuity in the $G_{0}(d)$ dependence between the wafers which we show can be attributed to the influence of barrier roughness. Perhaps not surprisingly, the mean thickness determined by TEM does not reflect the thickness relevant for electrical transport as indicated by the variation across the samples.

Figure 3 compares the barrier thickness $d$ and height $\varphi$ as determined by the BDR model for four different growth conditions and pretreatments. The trend of decreasing thickness and increasing barrier height seen across this series of samples corresponds to an increase in interface roughness as determined by TEM. The influence of roughness on the effective parameter values extracted using the BDR model has been discussed by Miller et al. ${ }^{19}$ We adopt this model and simulate roughness by assuming that the net conductance is the sum of parallel conductance channels with a Gaussian distribution of thicknesses with standard deviation $\sigma$. We take the sample series with the sharpest interface, the double etched sample, and use the mean extracted thickness of this series as a reference point. As shown in Fig. 3, we find that an increase in roughness of $\sigma=1 \AA(10 \%)$ and $\sigma=2 \AA(20 \%)$ can account for the apparent decrease in $d$ and increase in $\varphi$ for the degreased and single etched samples, respectively. The high roughness of the single etched samples is rein- forced by conducting AFM which shows the presence of high current, pinholelike features. ${ }^{12}$

In summary, we have grown $\mathrm{Co} / \sim 1.3 \mathrm{~nm} \mathrm{MgO} / \mathrm{InAs}$ trilayer structures with contact resistance values that are suitable for efficient spin injection/detection. The BDR and Stratton models have been used to determine whether tunneling dominates the conductance. A thermal smearing model gives further confirmation that tunneling dominates at all temperatures. Once the influence of roughness is taken into account, we find that the electrical properties of the barrier are relatively insensitive to surface pretreatment and growth temperature.

This work was supported by the UK-EPSRC Grant No. EP/C511972 and EP/C511980. We thank Professor R. Magnus for valuable advice.

${ }^{1}$ D. D. Awschalom and M. E. Flatte, Nat. Phys. 3, 153 (2007).

${ }^{2}$ H. C. Koo, H. Yi, J. B. Ko, J. Chang, S. H. Han, D. Jung, S. G. Huh, and J. Eom, Appl. Phys. Lett. 90, 022101 (2007).

${ }^{3}$ X. H. Lou, C. Adelmann, S. A. Crooker, E. S. Garlid, J. Zhang, K. S. M. Reddy, S. D. Flexner, C. J. Palmstrom, and P. A. Crowell, Nat. Phys. 3, 197 (2007).

${ }^{4}$ S. Datta and B. Das, Appl. Phys. Lett. 56, 665 (1990).

${ }^{5}$ X. Cartoixa, D. Z. Y. Ting, and Y. C. Chang, Appl. Phys. Lett. 83, 1462 (2003).

${ }^{6}$ A. Fert and H. Jaffres, Phys. Rev. B 6418, 184420 (2001).

${ }^{7}$ E. I. Rashba, Phys. Rev. B 62, R16267 (2000).

${ }^{8}$ G. Schmidt, D. Ferrand, L. W. Molenkamp, A. T. Filip, and B. J. van Wees, Phys. Rev. B 62, R4790 (2000).

${ }^{9}$ X. Jiang, R. Wang, R. M. Shelby, R. M. Macfarlane, S. R. Bank, J. S. Harris, and S. S. P. Parkin, Phys. Rev. Lett. 94, 056601 (2005).

${ }^{10}$ T. Kiyomura, Y. Maruo, and M. Gomi, J. Appl. Phys. 88, 4768 (2000).

${ }^{11}$ S. Mitani, T. Moriyama, and K. Takanashi, J. Appl. Phys. 93, 8041 (2003).

${ }^{12}$ L. J. Singh, R. A. Oliver, Z. H. Barber, D. A. Eustace, D. W. McComb, S. K. Clowes, A. M. Gilbertson, F. Magnus, W. R. Branford, L. F. Cohen, L. Buckle, P. D. Buckle, and T. Ashley, J. Phys. D 40, 3190 (2007).

${ }^{13}$ J. M. Rowell, in Tunneling Phenomena in Solids, edited by E. Burstein and S. Lundqvist (Plenum, New York, 1969), p. 273.

${ }^{14}$ W. F. Brinkman, R. C. Dynes, and J. M. Rowell, J. Appl. Phys. 41, 1915 (1970).

${ }^{15}$ R. Stratton, J. Phys. Chem. Solids 23, 1177 (1962).

${ }^{16}$ J. J. Akerman, J. M. Slaughter, R. W. Dave, and I. K. Schuller, Appl. Phys. Lett. 79, 3104 (2001), and references therein.

${ }^{17}$ B. Oliver and J. Nowak, J. Appl. Phys. 95, 546 (2004).

${ }^{18}$ L. Sheng, D. Y. Xing, and D. N. Sheng, Phys. Rev. B 70, 094416 (2004).

${ }^{19}$ C. W. Miller, Z. P. Li, J. Akerman, and I. K. Schuller, Appl. Phys. Lett. 90, 043513 (2007).

${ }^{20}$ C. W. Miller, Z. P. Li, I. K. Schuller, R. W. Dave, J. M. Slaughter, and J. Akerman, Phys. Rev. B 74, 212404 (2006).

${ }^{21}$ I. Vurgaftman, J. R. Meyer, and L. R. Ram-Mohan, J. Appl. Phys. 89, 5815 (2001).

${ }^{22}$ B. N. Murdin, K. Litvinenko, J. Allam, C. R. Pidgeon, M. Bird, K. Morrison, T. Zhang, S. K. Clowes, W. R. Branford, J. Harris, and L. F. Cohen, Phys. Rev. B 72, 085346 (2005).

${ }^{23}$ C. H. Moller, O. Kronenwerth, D. Grundler, W. Hansen, C. Heyn, and D. Heitmann, Appl. Phys. Lett. 80, 3988 (2002).

${ }^{24}$ J. G. Simmons, J. Appl. Phys. 34, 1793 (1963).

${ }^{25}$ J. J. Akerman, I. V. Roshchin, J. M. Slaughter, R. W. Dave, and I. K. Schuller, Europhys. Lett. 63, 104 (2003).

${ }^{26}$ Y. Bugoslavsky, Y. Miyoshi, S. K. Clowes, W. R. Branford, M. Lake, I. Brown, A. D. Caplin, and L. F. Cohen, Phys. Rev. B 71, 104523 (2005). 